\title{
No advantage of time-of-flight magnetic resonance angiography at 3 Tesla compared to 1.5 Tesla in the follow-up after endovascular treatment of cerebral aneurysms
}

\author{
Jan-Hendrik Buhk • Kai Kallenberg • \\ Alexander Mohr • Peter Dechent • Michael Knauth
}

Received: 19 February 2008 / Accepted: 6 May 2008 / Published online: 4 June 2008

(C) The Author(s) 2008

\begin{abstract}
Introduction Long-term follow-up after coil embolization of intracranial aneurysms is mandatory to monitor coil compacting and aneurysm recurrence. Most centers perform one digital subtraction angiography (DSA) on follow-up continuing with time-of-flight magnetic resonance angiography (TOF-MRA). This study explores the diagnostic value of TOF-MRA at $1.5 \mathrm{~T}$ versus $3 \mathrm{~T}$ compared to DSA. Materials and methods In 18 patients with 20 aneurysms treated with coil embolization, TOF-MRA at 1.5 and $3 \mathrm{~T}$ were performed the day before follow-up DSA, the latter serving as reference. Optimized diagnostic protocols were applied $(1.5 \mathrm{~T}: 0.78 \times 0.55 \times 0.8 \mathrm{~mm}$, voxel size; acquisition time (TA), $6.37 \mathrm{~min} ; 3 \mathrm{~T}: 0.56 \times 0.45 \times 0.65 \mathrm{~mm}$, voxel size; TA, $3.12 \mathrm{~min}$ ). Three independent neuroradiologists experienced in neuroendovascular therapy rated the occlusion rate ("complete occlusion" vs. "residual neck" vs. "residual aneurysm") and compared the two methods subjectively. Weighted $\kappa$ statistics were calculated to assess the level of interobserver agreement.

Results Compared to DSA, TOF-MRA was more sensitive in detecting neck remnants, with a slight advantage at $3 \mathrm{~T}$. Regarding artifact load, there are advantages at $1.5 \mathrm{~T}$. Ratings of the occlusion rate correlated highly between all
\end{abstract}

J.-H. Buhk $(\bowtie) \cdot$ K. Kallenberg $\cdot$ A. Mohr $\cdot$ M. Knauth Department of Neuroradiology, University of Göttingen, Robert-Koch-Str. 40,

37075 Goettingen, Germany

e-mail: jbuhk@gwdg.de

J.-H. Buhk $\cdot$ K. Kallenberg $\cdot$ P. Dechent

MR-Research in Neurology and Psychiatry,

University of Göttingen,

Goettingen, Germany observers $(r>0.85, p<0.001$, respectively). Interobserver agreement was high in all cases ( $\kappa_{\mathrm{w}} \approx 0.8$, respectively). Conclusion TOF-MRA is a reliable tool for follow-up imaging of cerebral aneurysms after endovascular treatment. Our study shows no advantage of TOF-MRA at $3 \mathrm{~T}$ over $1.5 \mathrm{~T}$, when comparable measurement protocols are applied. TOF-MRA at $1.5 \mathrm{~T}$ therefore provides appropriate information regarding a therapeutic decision.

Keywords Aneurysm · GDC coiling · Follow-up · Time-of-flight angiography $\cdot$ MR angiography $\cdot 3$ Tesla

\section{Introduction}

Endovascular treatment of intracranial aneurysms today is a widespread and approved technique, and in a majority of cases, an alternative to the neurosurgical approach [1-3]. However, recanalization of a cerebral aneurysm treated with endovascular coil embolization occurs in up to $30 \%$ of all patients and carries a small risk of rebleeding [1-5]. Most neurointerventional centers therefore currently perform a post-interventional follow-up digital subtraction angiography (DSA) after 6 months. If there is a good correlation with a magnetic resonance angiography (MRA) obtained on the same occasion, follow-up is continued by MRA at regular intervals. With regard to current publications, it is discussed controversially whether TOF-MRA or contrast-enhanced MRA (CE-MRA) is the superior technique for aneurysm follow-up [6,7]. Other authors recommend contrastenhanced TOF-MRA to enhance the sensitivity $[8,9]$.

The purpose of our prospective study was to find out whether there are significant advantages in performing TOF-MRA at $3 \mathrm{~T}$ as compared to $1.5 \mathrm{~T}$ in a routine 
diagnostic setting. The study was performed against the background of an increasing number of 3 T MR scanners used in routine diagnostic imaging worldwide [10]. Therefore, we used optimized but comparable diagnostic sequences that should be easily reproducible at any MR scanner.

\section{Materials and methods}

Patients and aneurysms

Eighteen patients with 20 aneurysms treated with endovascular coil embolization in our department from 2004 until 2006 (nine men; median age, 61 years; range, 29-78 years) participated in the prospective study. Aneurysm locations were as follows: anterior communicating artery (Acom), $n=8$; internal carotid artery (ICA), $n=3$; posterior communicating artery (Pcom), $n=4$; middle cerebral artery (MCA), $n=1$; and basilar artery (BA), $n=4$. The coils used are presented in Table 1. Stent remodeling was performed in eight patients $\left(7 \times\right.$ Neuroform $3^{\mathrm{TM}}$, Boston Scientific, Natick, MA, USA; $1 \times$ MicroDriver ${ }^{\mathrm{TM}}$, Medtronic, Minneapolis, MN, USA). Approval of the local ethics committee and informed patient consent were obtained.

\section{Technique}

All patients were admitted for standard 6-months follow-up DSA, which included a rotational acquisition (AxiomArtis dBA, Siemens Medical Solutions, Forchheim, Germany). TOF-MRA were always performed the day before the DSA exam.

TOF-MRA at $3 \mathrm{~T}$ (Magnetom Trio, Siemens Medical Solutions, Erlangen, Germany; eight-channel-phased array head coil) was performed with the following parameters: repetition time (TR), $23 \mathrm{~ms}$; echo time (TE), $3.68 \mathrm{~ms}$; image matrix, 448; field of view (FOV), $170 \times 180 \mathrm{~mm}$ (effective voxel size $=0.56 \times 0.45 \times 0.65 \mathrm{~mm}$ ); flip angle, $18^{\circ}$; parallel acquisition technique (PAT, generalized autocalibrating partially parallel acquisitions, GRAPPA) factor, 2; and TA, $3.12 \mathrm{~min}$.

TOF-MRA at $1.5 \mathrm{~T}$ (Magnetom Sonata, Siemens Medical Solutions, Erlangen, Germany; CP head coil) was

Table 1 Range of coils used, divided by manufacturer and bioactive coating.

\begin{tabular}{llll}
\hline & Bare platinum & Bioactive & Both \\
\hline Micrus endovascular $^{\mathrm{a}}$ & 2 & 2 & 1 \\
BostonScientific & 7 & 6 & 1 \\
Both & - & - & 1 \\
Total & 9 & 8 & 3 \\
\hline
\end{tabular}

${ }^{\mathrm{a}}$ Renens, Switzerland performed with the following parameters: $\mathrm{TR}=35 \mathrm{~ms}$; $\mathrm{TE}=$ $4.72 \mathrm{~ms}$; image matrix, 384; FOV, $190 \times 210 \mathrm{~mm}$ (effective voxel size $=0.78 \times 0.55 \times 0.80 \mathrm{~mm}$ ); flip angle, $25^{\circ}$; and TA, $6.37 \mathrm{~min}$ (no PAT).

Image postprocessing

TOF-MRA data were viewed as three-dimensional (3D) maximum-intensity projection (MIP) images in free directions as well as multiplanar reformatted (MPR) thin-slice images. DSA data included 2D series as well as 3D rotational angiography (3D-RA)/angiographic computed tomography images in $3 \mathrm{D}$ volume-rendering technique, 3D MIP, and thin-slice MPR. All image viewing was performed on an OsiriX ${ }^{\mathrm{TM}}$ workstation [11].

Statistical analysis

Three experienced neuroradiologists (K.K., A.M., and M.K.) independently performed the image viewing and rating. Firstly, the state of aneurysm occlusion was rated following a three-step ordinal scale ("complete occlusion" vs. "residual neck" vs. "residual aneurysm") [3]. Pearson's correlation coefficient was calculated for each pair of methods in the diagnostic rating. Weighted $\kappa$ statistics were calculated to assess interobserver variabilities; a value above $\kappa=0.6$ was assumed to represent substantial agreement $[12,13]$. Secondly, a direct method-comparison questionnaire to achieve a statement of diagnostic superiority vs. equivalence vs. inferiority was completed by each observer. A similar questionnaire was completed regarding the artifact load.

\section{Results}

Occlusion rate

The cumulative results of the diagnostic viewing performed by all three raters regarding the occlusion rate are presented in Table 2. Both 1.5 and $3 \mathrm{~T}$ TOF-MRA were more sensitive than DSA in detecting a neck remnant (14/60 and $16 / 60$ vs. $10 / 60$, respectively). The results regarding the

Table 2 Cumulative results of the aneurysm occlusion ratings by three observers (20 aneurysms, therefore 60 ratings).

\begin{tabular}{lccc}
\hline & DSA & $\begin{array}{c}\text { TOF-MRA } \\
\text { at } 1.5 \mathrm{~T}\end{array}$ & $\begin{array}{c}\text { TOF-MRA } \\
\text { at } 3 \mathrm{~T}\end{array}$ \\
\hline Complete occlusion & 40 & 35 & 33 \\
Residual neck & 10 & 14 & 16 \\
Residual aneurysm & 10 & 11 & 11 \\
Overall & 60 & 60 & 60 \\
\hline
\end{tabular}


detection of residual aneurysms are nearly the same for all three methods (11/60 and $11 / 60$ vs. $10 / 60$, respectively). A consensus rating was not performed. Ratings of the occlusion rate correlated highly between all observers $(r>0.85$ and $p<0.001$, respectively).

Table 3 shows the results of the $\kappa$ statistics regarding the occlusion rate. All observers were tested against each other regarding every method. Interobserver agreement is very good for all groups $\left(\kappa_{\mathrm{w}}>0.8\right)[12,13]$. The distribution of the results in between the observer pairs is homogenous.

\section{Direct comparison}

Firstly, a direct comparison of the diagnostic value of each technique against the other was asked. The cumulative results are presented graphically in Fig. 1 and show no trend toward either field strength. Secondly, a direct comparison of the artifact load was asked. The cumulative results are displayed in Fig. 2. In only four out of 60 ratings, TOF-MRA at $3 \mathrm{~T}$ was scored to be less affected by artifacts as TOF-MRA at $1.5 \mathrm{~T}$. In contrast, in 20 aneurysms, TOF-MRA at $1.5 \mathrm{~T}$ was scored superior. That trend was coherent when comparing bare platinum versus bioactive coil packages (subgroup data not shown).

Possible sources of artifacts are presented in the case figures. A small coil package and a displaced coil cause no relevant artifact (Fig. 3c,d). A larger coil package causes impaired flow signal in the adjacent vessel segments (Figs. 4c and d and 5c and d, closed arrows) but does not obscure a small neck remnant (Fig. $4 \mathrm{c}$ and d, open arrows). The artifacts caused by a nitinol stent (case shown: Neuroform $3^{\mathrm{TM}}$, BostonScientific) are acceptable in most of the cases and only insignificantly pronounced at $3 \mathrm{~T}$ (Fig. 5c and d, open arrow).

\section{Discussion}

An aneurysm recurrence rate between $20 \%$ and $30 \%$ after endovascular treatment has been reported [3, 4, 14, 15]. The re-treatment rate in the International Subarachnoid Aneurysm Trial (ISAT) study group was 17.4\% [14]. As currently no guideline exists proposing MRA as the follow-

Table 3 Interobserver agreement, measured as $k_{\mathrm{w}}$, achieves substantially high agreement for all techniques.

\begin{tabular}{lccc}
\hline & DSA & $\begin{array}{c}\text { TOF-MRA } \\
\text { at } 1.5 \mathrm{~T}\end{array}$ & $\begin{array}{c}\text { TOF-MRA } \\
\text { at } 3 \mathrm{~T}\end{array}$ \\
\hline Observers 1 vs. 2 & 0.81 & 0.74 & 0.72 \\
Observers 1 vs. 3 & 0.78 & 0.86 & 0.78 \\
Observers 2 vs. 3 & 0.87 & 0.81 & 0.82 \\
Mean $\kappa_{\mathrm{w}}$ & 0.82 & 0.80 & 0.78 \\
\hline
\end{tabular}

diagnostic value: TOF-MRA at $1.5 \mathrm{~T}$ vs. $3 \mathrm{~T}$

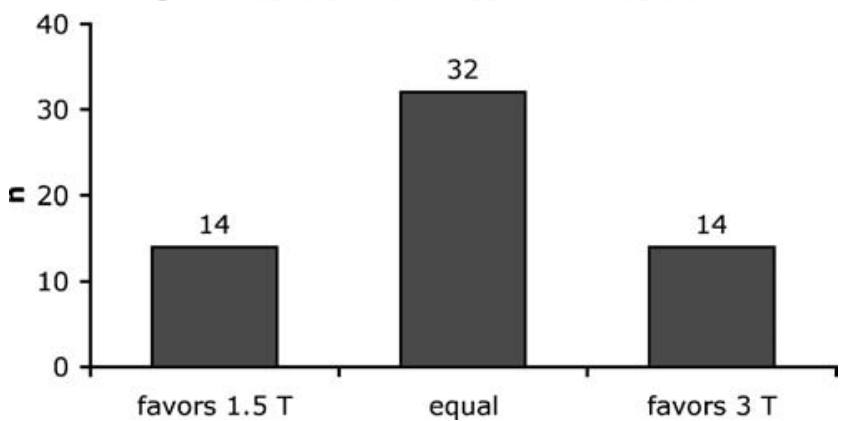

Fig. 1 The diagram shows the cumulative results (three observers and 20 aneurysms, therefore, 60 ratings) of the method comparison regarding the diagnostic value of TOF-MRA at 1.5 vs. $3 \mathrm{~T}$

up method of the first choice, the gold standard in followup diagnostic imaging is still the intraarterial DSA, associated with an inherent small rate of permanent neurological deficit [16]. Therefore, in search of an aneurysm recurrence after endovascular treatment, the most sensitive and least invasive imaging should be performed. It has been shown that TOF-MRA is a sensitive and safe technique regarding coiled aneurysms; some authors have concluded that it may be even more sensitive than DSA [5, 9, 17-21]. Others recommend CE-MRA or contrastenhanced TOF-MRA to raise sensitivity regarding residual filling and to minimize stent-related artifacts $[6,7,9,22$, 23]. In our relatively small collective, more neck remnants were detected in TOF-MRA than in DSA. However, that finding has no relevance with respect to therapeutical consequences: A neck remnant would not be treated right away but rather followed up more closely - by TOF-MRAand treated when evolving into a real aneurysm recurrence. If all follow-up imaging were performed by DSA, the approach would be the same.

Our study revealed no obvious advantage of TOF-MRA at $3 \mathrm{~T}$ over $1.5 \mathrm{~T}$ regarding the diagnostic value of depicting aneurysm recurrence. There are technical advantages at $3 \mathrm{~T}$, as there is the much shorter acquisition time due to higher signal-to-noise ratio and application of parallel imaging

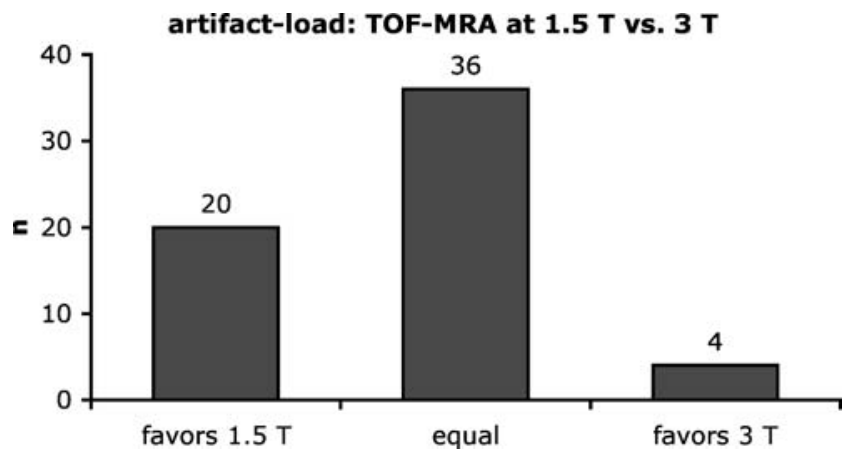

Fig. 2 The diagram shows the cumulative results (three observers and 20 aneurysms, therefore, 60 ratings) of the method comparison regarding the artifact load of TOF-MRA at 1.5 vs. $3 \mathrm{~T}$ 
Fig. 3 Follow-up imaging of a 65 -year-old male patient with a history of subarachnoid hemorrhage $(\mathrm{SAH})$ and endovascular therapy of an Acom aneurysm. Selective DSA reveals coil compacting and a residual aneurysm (a unsubtracted, b subtracted). A stretched coil is located in the ICA (a arrows). TOF-MRA (thick-slice MIP and slice thickness $5 \mathrm{~mm}$, respectively) shows the same finding regardless of the field strength $(\mathbf{c} 1.5 \mathrm{~T}$ and $\mathbf{d} 3 \mathrm{~T})$. No artifacts occur due to the dislocated coil
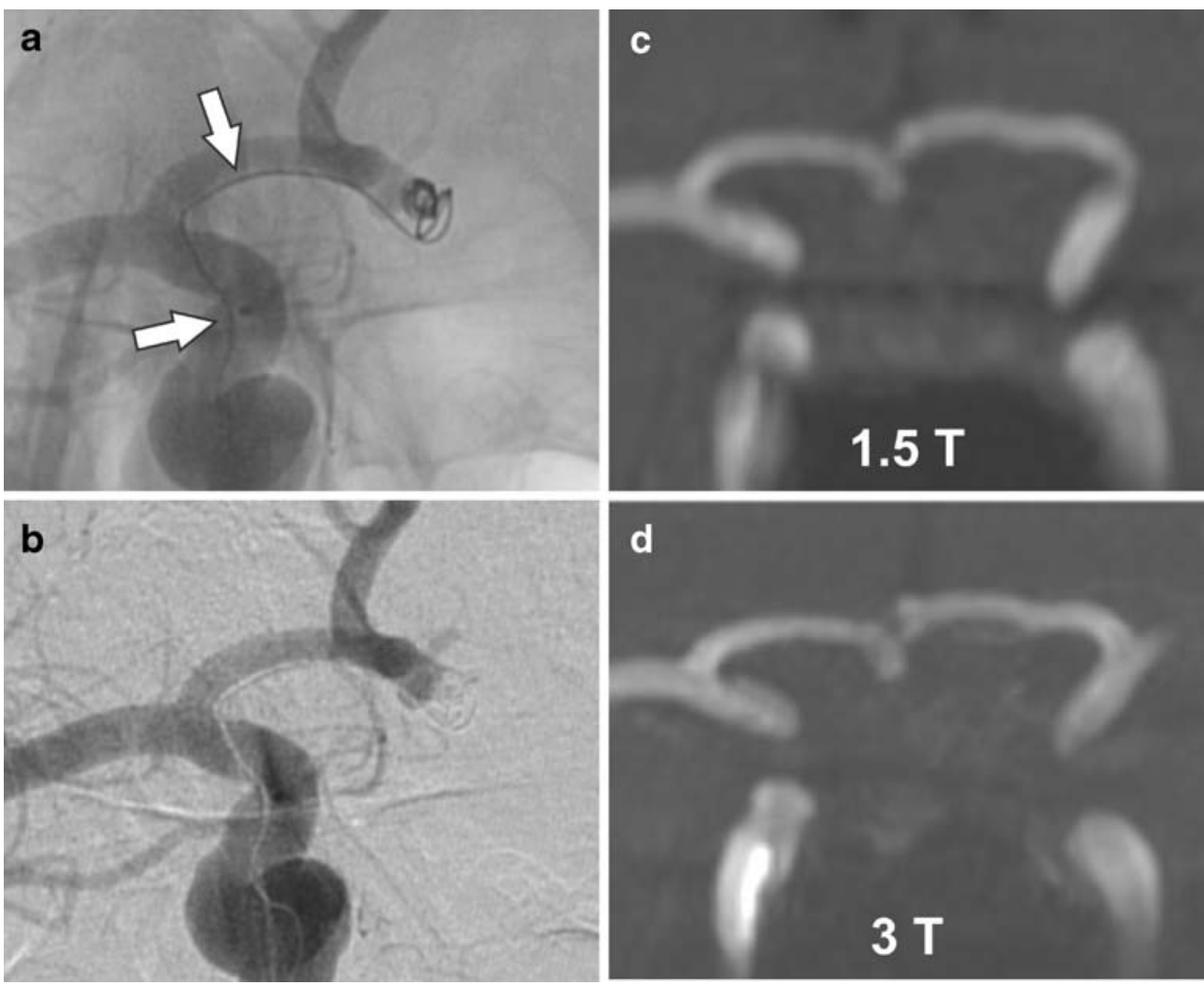
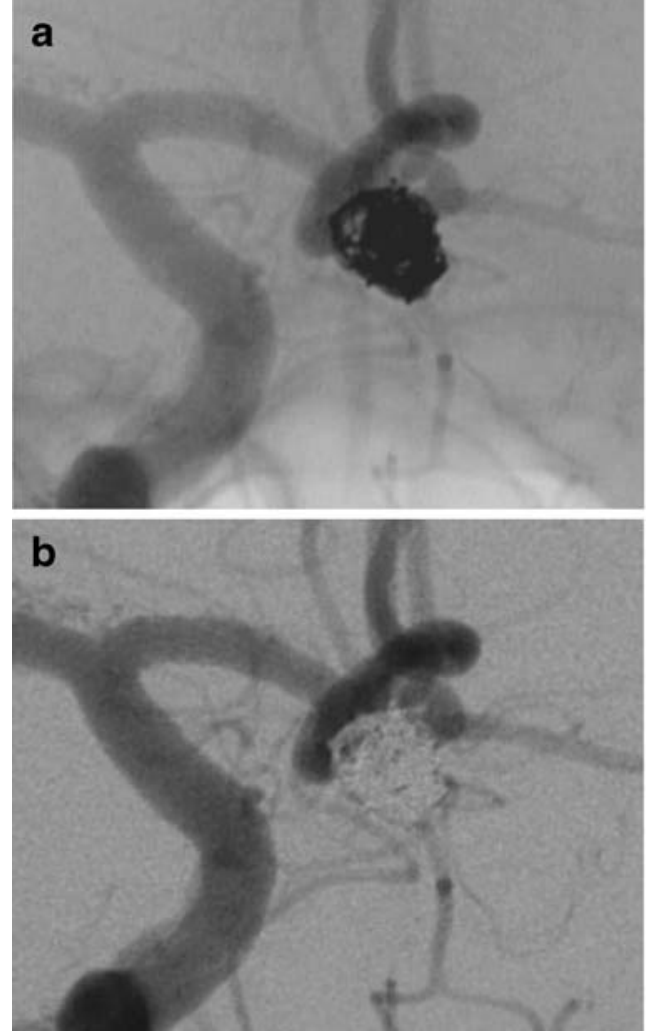

Fig. 4 Follow-up imaging of a 59-year-old male patient with a history of SAH and endovascular therapy of an Acom aneurysm. The DSA exam was rated as complete occlusion by all observers (a unsubtracted and b subtracted). TOF-MRA (thick-slice MIP and slice thickness
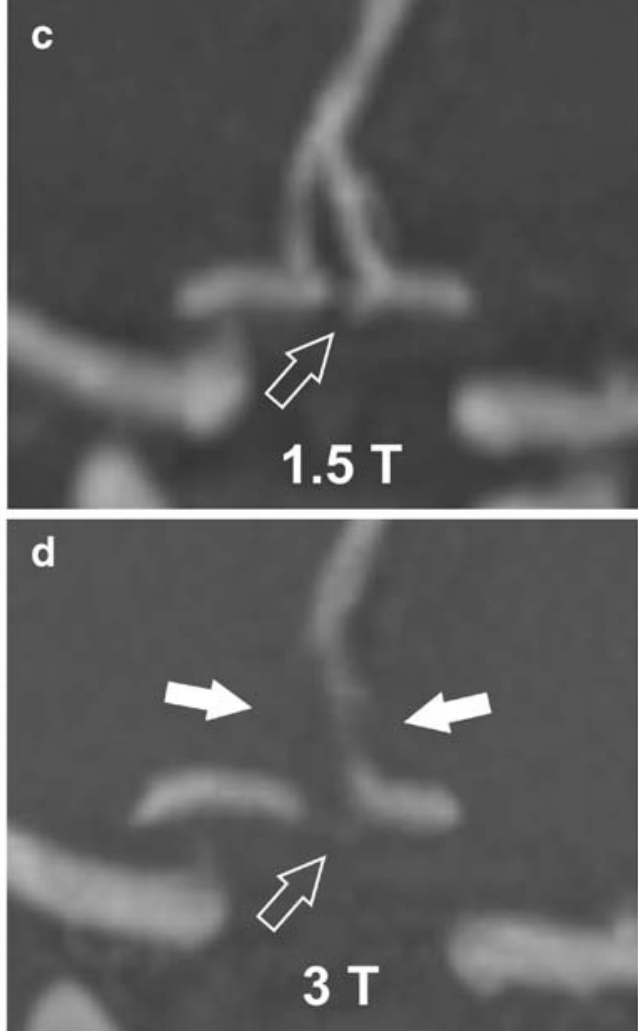

$10 \mathrm{~mm}$, respectively) revealed a very small neck remnant needing no re-treatment (c $1.5 \mathrm{~T}$; d $3 \mathrm{~T}$, open arrows). Note the coil-related artifacts in the TOF-MRA at $3 \mathrm{~T}$ simulating a stenosis of the left and actually an occlusion of the right A2 segment (d closed arrows) 

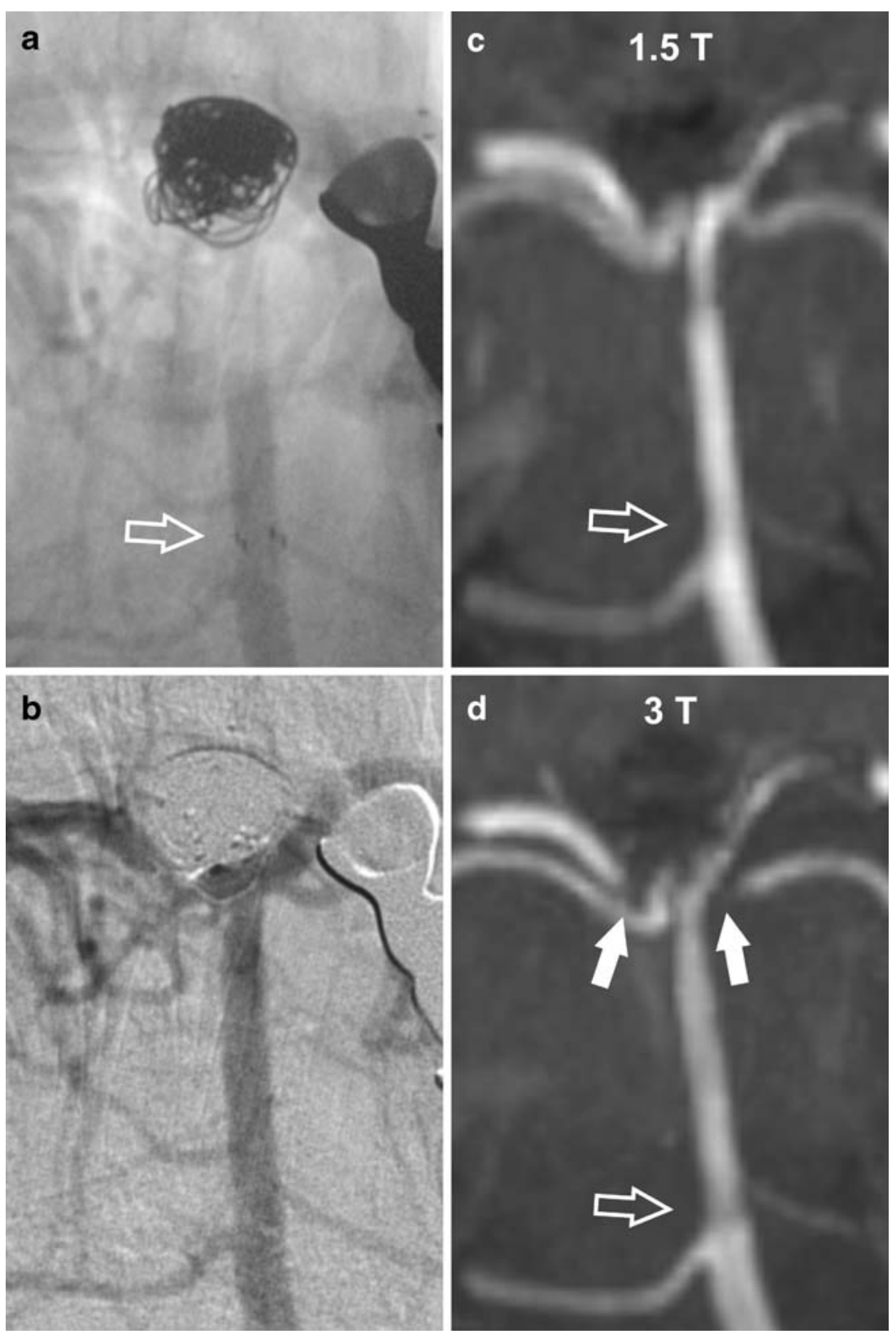

Fig. 5 Follow-up imaging of a 32-year-old male patient with a history of SAH and stent-protected endovascular therapy of a large basilar tip aneurysm. The DSA exam shows some coil compacting but no refilling (a unsubtracted and $\mathbf{b}$ subtracted). That confirms the TOFMRA results (c $1.5 \mathrm{~T}$, d $3 \mathrm{~T}$; thick-slice MIP and slice thickness,

techniques. A higher spatial resolution at a shorter acquisition time was achieved in the present study applying $3 \mathrm{~T}$, potentially explaining the additional two neck remnants detected ( $3 \mathrm{~T}=16$ vs. $1.5 \mathrm{~T}=14)$. That was possible despite the more pronounced susceptibility artifacts at $3 \mathrm{~T}$. The most important diagnostic impairment in our collective results from an artificial narrowing of the lumen of adjacent vessels at $3 \mathrm{~T}$, like that shown earlier in vivo and in vitro (see Figs. 4d and 5d) [9, 24].
$10 \mathrm{~mm}$, respectively). Again, the visualization of the vessels adjacent to the coil package is impaired at $3 \mathrm{~T}$ (d closed arrows). Regardless of the field strength, only minor artifacts occur due to the Neuroform ${ }^{\mathrm{TM}}$ stent (a, c, and $\mathbf{d}$ open arrows)

The small subgroup analysis regarding the coil type did not reveal any differences - the study collective may be too small. Performing further subgroup analyses regarding the aneurysm size - and therefore, the amount of implant material - and the influence of stent-remodeling on the artifact load would be interesting, especially because of the observation of less pronounced artifacts with only few implant material (see Fig. 3). Due to the relatively small study collective, we did not perform such a subgroup analysis. 
However, TOF-MRA at $1.5 \mathrm{~T}$ in our study was still more sensitive in depicting neck remnants than DSA, the current gold standard. The acquisition time of $6.37 \mathrm{~min}$ at $1.5 \mathrm{~T}$ is acceptable in clinical neuroimaging if the focus lies on the cerebral vasculature.

Magnetic field interactions at $3 \mathrm{~T}$ have been not examined for all intracranial implants yet, e.g., some older aneurysm clips are known not to be suitable for magnetic resonance imaging (MRI) at $3 \mathrm{~T}$. On the other hand, the Neuroform $^{\mathrm{TM}}$ stent and all platinum coils are [25-27]. Therefore, the group of patients not suitable for $3 \mathrm{~T}$ is relatively small. However, if in doubt regarding the implant situation, lower field strength should be preferred.

Our results support the conclusions of Urbach et al. regarding the possibility of only performing DSA if changes in aneurysm configuration are observed at follow-up TOFMRA [5].

\section{Conclusion}

The key findings of our study are:

1. TOF-MRA was not inferior compared to DSA and might therefore replace DSA as the standard method for aneurysm follow-up.

2. A comparable sequence at $3 \mathrm{~T}$ did not translate into diagnostic benefit compared to $1.5 \mathrm{~T}$.

Conflict of interest statement We declare that we have no conflict of interest.

Open Access This article is distributed under the terms of the Creative Commons Attribution Noncommercial License which permits any noncommercial use, distribution, and reproduction in any medium, provided the original author(s) and source are credited.

\section{References}

1. Byrne JV, Sohn MJ, Molyneux AJ, Chir B (1999) Five-year experience in using coil embolization for ruptured intracranial aneurysms: outcomes and incidence of late rebleeding. J Neurosurg 90:656-663

2. Molyneux A, Kerr R, Stratton I et al (2002) International Subarachnoid Aneurysm Trial (ISAT) of neurosurgical clipping versus endovascular coiling in 2143 patients with ruptured intracranial aneurysms: a randomised trial. Lancet 360:1267-1274

3. Raymond J, Guilbert F, Weill A et al (2003) Long-term angiographic recurrences after selective endovascular treatment of aneurysms with detachable coils. Stroke 34:1398-1403

4. Ries T, Siemonsen S, Thomalla G, Grzyska U, Zeumer H, Fiehler J (2007) Long-term follow-up of cerebral aneurysms after endovascular therapy prediction and outcome of retreatment. AJNR Am J Neuroradiol 28:1755-1761

5. Urbach H, Dorenbeck U, von Falkenhausen M et al (2008) Threedimensional time-of-flight MR angiography at $3 \mathrm{~T}$ compared to digital subtraction angiography in the follow-up of ruptured and coiled intracranial aneurysms: a prospective study. Neuroradiology (in press)

6. Gibbs GF, Huston J III, Bernstein MA, Riederer SJ, Brown RDJ (2005) 3.0-Tesla MR angiography of intracranial aneurysms: comparison of time-of-flight and contrast-enhanced techniques. J Magn Reson Imaging 21:97-102

7. Pierot L, Delcourt C, Bouquigny F et al (2006) Follow-up of intracranial aneurysms selectively treated with coils: prospective evaluation of contrast-enhanced MR angiography. AJNR Am J Neuroradiol 27:744-749

8. Cottier JP, Bleuzen-Couthon A, Gallas S et al (2003) Intracranial aneurysms treated with Guglielmi detachable coils: is contrast material necessary in the follow-up with 3D time-of-flight MR angiography? AJNR Am J Neuroradiol 24:1797-1803

9. Majoie CB, Sprengers ME, van Rooij WJ et al (2005) MR angiography at $3 \mathrm{~T}$ versus digital subtraction angiography in the follow-up of intracranial aneurysms treated with detachable coils. AJNR Am J Neuroradiol 26:1349-1356

10. Lu H, Nagae-Poetscher LM, Golay X, Lin D, Pomper M, van Zijl PC (2005) Routine clinical brain MRI sequences for use at 3.0 Tesla. J Magn Reson Imaging 22:13-22

11. Rosset A, Spadola L, Ratib O (2004) OsiriX: an open-source software for navigating in multidimensional DICOM images. J Digit Imaging 17:205-216

12. Cohen J (1968) Weighted kappa: nominal scale agreement with provision for scaled disagreement or partial credit. Psychol Bull 70:213-220

13. Landis JR, Koch GG (1977) The measurement of observer agreement for categorical data. Biometrics 33:159-174

14. Campi A, Ramzi N, Molyneux AJ et al (2007) Retreatment of ruptured cerebral aneurysms in patients randomized by coiling or clipping in the International Subarachnoid Aneurysm Trial (ISAT). Stroke 38:1538-1544

15. Piotin M, Spelle L, Mounayer C et al (2007) Intracranial aneurysms: treatment with bare platinum coils - aneurysm packing, complex coils, and angiographic recurrence. Radiology 243: $500-508$

16. Willinsky RA, Taylor SM, TerBrugge K, Farb RI, Tomlinson G, Montanera W (2003) Neurologic complications of cerebral angiography: prospective analysis of 2,899 procedures and review of the literature. Radiology 227:522-528

17. Brunereau L, Cottier JP, Sonier CB et al (1999) Prospective evaluation of time-of-flight MR angiography in the follow-up of intracranial saccular aneurysms treated with Guglielmi detachable coils. J Comput Assist Tomogr 23:216-223

18. Anzalone N, Righi C, Simionato F et al (2000) Three-dimensional time-of-flight MR angiography in the evaluation of intracranial aneurysms treated with Guglielmi detachable coils. AJNR Am J Neuroradiol 21:746-752

19. Boulin A, Pierot L (2001) Follow-up of intracranial aneurysms treated with detachable coils: comparison of gadolinium-enhanced 3D time-of-flight MR angiography and digital subtraction angiography. Radiology 219:108-113

20. Yamada N, Hayashi K, Murao K, Higashi M, Iihara K (2004) Time-of-flight MR angiography targeted to coiled intracranial aneurysms is more sensitive to residual flow than is digital subtraction angiography. AJNR Am J Neuroradiol 25:11541157

21. Deutschmann HA, Augustin M, Simbrunner J et al (2007) Diagnostic accuracy of 3D time-of-flight MR angiography 
compared with digital subtraction angiography for follow-up of coiled intracranial aneurysms: influence of aneurysm size. AJNR Am J Neuroradiol 28:628-634

22. Gauvrit JY, Leclerc X, Caron S, Taschner CA, Lejeune JP, Pruvo JP (2006) Intracranial aneurysms treated with Guglielmi detachable coils: imaging follow-up with contrast-enhanced MR angiography. Stroke 37:1033-1037

23. Lovblad KO, Yilmaz H, Chouiter A et al (2006) Intracranial aneurysm stenting: follow-up with MR angiography. J Magn Reson Imaging 24:418-422

24. Walker MT, Tsai J, Parish T et al (2005) MR angiographic evaluation of platinum coil packs at $1.5 \mathrm{~T}$ and $3 \mathrm{~T}$ : an in vitro assessment of artifact production: technical note. AJNR Am J Neuroradiol 26:848-853

25. Shellock FG (2002) Biomedical implants and devices: assessment of magnetic field interactions with a 3.0-Tesla MR system. J Magn Reson Imaging 16:721-732

26. Shellock FG, Tkach JA, Ruggieri PM, Masaryk TJ, Rasmussen PA (2003) Aneurysm clips: evaluation of magnetic field interactions and translational attraction by use of "long-bore" and "short-bore" 3.0-T MR imaging systems. AJNR Am J Neuroradiol 24:463-471

27. Nehra A, Moran CJ, Cross DT, Derdeyn CP (2004) MR safety and imaging of neuroform stents at 3T. AJNR Am J Neuroradiol 25:1476-1478 